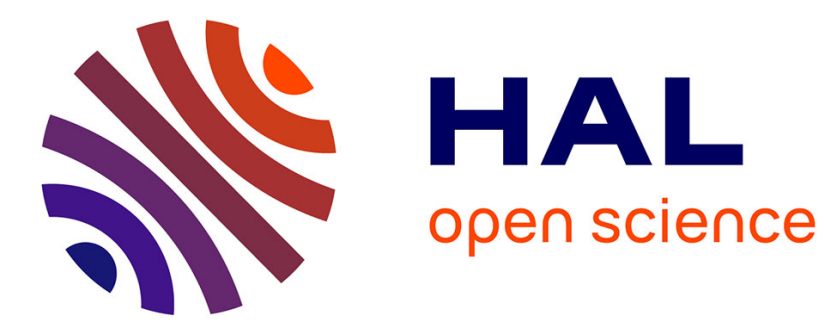

\title{
Visible-transparent and UV/IR-opaque colloidal dispersions of Ga-doped zinc oxide nanoparticles
}

Isabelle Trenque, Manuel Gaudon, Etienne Duguet, Stéphane Mornet

\section{To cite this version:}

Isabelle Trenque, Manuel Gaudon, Etienne Duguet, Stéphane Mornet. Visible-transparent and UV/IR-opaque colloidal dispersions of Ga-doped zinc oxide nanoparticles. New Journal of Chemistry, 2016, 40 (8), pp.7204-7209. 10.1039/C6NJ01668E . hal-01357073

\section{HAL Id: hal-01357073 https://hal.science/hal-01357073}

Submitted on 18 Jan 2021

HAL is a multi-disciplinary open access archive for the deposit and dissemination of scientific research documents, whether they are published or not. The documents may come from teaching and research institutions in France or abroad, or from public or private research centers.
L'archive ouverte pluridisciplinaire HAL, est destinée au dépôt et à la diffusion de documents scientifiques de niveau recherche, publiés ou non, émanant des établissements d'enseignement et de recherche français ou étrangers, des laboratoires publics ou privés. 


\section{Visible-transparent and UV/IR-opaque colloidal dispersions of Ga- doped zinc oxide nanoparticles}

Received 00th January 20xx, Accepted 00th January 20xx

DOI: $10.1039 / \times 0 \times x 00000 x$

www.rsc.org/

\author{
I Trenque, ${ }^{a, b}$ M. Gaudon, ${ }^{a, b}$ E. Duguet ${ }^{a, b}$ and S. Mornet ${ }^{a, b *}$
}

Preliminary Mie scattering calculations show that $\mathrm{ZnO}:$ Ga nanoparticles dispersed in a visible-transparent matrix could create an efficient infrared barrier while remaining transparent to visible light. The $\mathrm{ZnO}: \mathrm{Ga}$ supracolloidal particles elaborated by polyol process are successfully disaggregated in the presence of an alkanethiol leading to stable dispersions whose absorption spectra are in agreement with calculations.

\section{Introduction}

Thermal insulating coatings for window glasses which are able to limit the infrared transmission whereas the visible transmittance remains high, are of great interest, as illustration in building insulation. Transparent conductive oxides (TCOs) can be used as such selective visible light filters. Doping $\mathrm{ZnO}$ oxide matrix with n-type donors ( $\mathrm{Al}, \mathrm{Ga}, \mathrm{In}, \mathrm{F}$ ) is the most developed route to produce these kinds of heat mirrors. ${ }^{1}$ Our own recent results demonstrated that aluminum or gallium-doped $\mathrm{ZnO}$ are promising candidates for getting efficient infrared absorbing powders. ${ }^{2}$ Two kinds of devices may be engineered: dense and thin films, ${ }^{3}$ or more original systems such as varnishes and coatings added or spread to the glazing in which TCO nanoparticles are dispersed in a glass or polymeric matrix. ${ }^{4}$ At the present time, these films are produced by physical processes, e.g. magnetron sputtering, CVD, spin coating and dip coating, but these techniques are expensive and complicated to extend on large surfaces and at industrial scale. An alternative approach consists in the application of varnish or the laying of polymeric films where would be dispersed particles transparent in the visible range and absorbing/diffusing in the infrared one. Nevertheless, the refractive index of $\mathrm{ZnO}(\mathrm{n}=2.00)$ remains too high for easily ensuring a sufficient transmission of the visible light through the envisaged films/varnishes systems. Indeed, diffusion at the particle/matrix interface leads to a drastic decrease of the visible transparency. One way to increase the transparency is the achievement of dispersions based on nanoscaled particles (colloidal dispersions).

In this study, theoretical calculations of the transmission of nano-colloidal dispersions and thin films were first performed and compared, showing that nano-colloidal varnishes/paints, i.e. when the size of the dispersed doped $\mathrm{ZnO}$ particles is lower

\footnotetext{
a. CNRS, ICMCB, UPR 9048, 33600 Pessac, France. Fax:+33540002761; Tel:

+33540006685; E-mail: mornet@icmcb-bordeaux.cnrs.fr
}

b. Univ. Bordeaux, ICMCB, UPR 9048, 33600 Pessac, France. than $25 \mathrm{~nm}$, can be suitable alternatives to thin films. Then, a synthesis method of nano-colloidal dispersions with high visible transparency and UV/IR opaque behavior was proposed.

\section{Experimental}

Synthesis of the Ga-doped ZnO particles by the polyol route.

ZnO:Ga particles were prepared by the polyol-mediated precipitation route that consists in the forced hydrolysis of zinc acetate and gallium salt in diethylene glycol (DEG) at high temperature according to the procedure previously described. ${ }^{2}$ In a typical procedure, zinc acetate dihydrate (Sigma, purity $\geq 99 \%$ ) was first dissolved in DEG ( $99 \%$ Sigma). The gallium source (Solvay, acid solution of gallium nitrate, Ga: $128 \mathrm{~g} / \mathrm{L}$, $\left[\mathrm{H}^{+}\right]=0.29 \mathrm{M}$ ) was added to the raw mixture in a round flask equipped with a condenser. The cation metal concentration in DEG was $0.1 \mathrm{M}$ (about $8 \mathrm{~g} \cdot \mathrm{L}^{-1}$ ). The temperature of the reactive medium was increased to $170^{\circ} \mathrm{C}$ for $1 \mathrm{~h}$ under continuous stirring.

\section{Post-functionalization with hexadecanethiol.}

Typically, $2 \mathrm{~mL}$ of the previous dispersion were mixed with 2 $\mathrm{mL}$ of ethanol and $2 \mathrm{~mL}$ of $0.39 \mathrm{M}$ solution of hexadecane-1thiol (HDT, Sigma $\geq 95 \%$ ) in ethanol, because of the nonsolubility of HDT in DEG. The mixture was vigorously homogenized with a vortex mixer for several minutes. The solubilization of HDT in ethanol was improved by heating up the mixture at $40^{\circ} \mathrm{C}$. After overnight incubation, the nanoparticles were recovered by centrifugation, washed several times with ethanol and then dispersed in $2 \mathrm{~mL}$ of chloroform $\left(\mathrm{CHCl}_{3}\right.$, Sigma-Aldrich, $\left.\geq 99.5 \%\right)$ under $15 \mathrm{~min}$ sonication in an ultrasonic bath. In order to discriminate the effects of dispersion and solubilization of particles observed by UV/vis spectroscopy, we used acetic acid as control. The effect of dissolution by acetic acid is directly investigated in the asprepared dispersion, for which a defined concentration of $\mathrm{ZnO}$ 
particles can be adjusted, by adding $2 \mathrm{ml}$ of glacial acetic acid (Sigma-Aldrich, $\geq 99 \%$ ) dissolved in $2 \mathrm{~mL}$ of DEG.

\section{Characterization of the Ga-doped ZnO particles/dispersions.}

The morphology and aggregation state of the particles were observed by electron microscopy. The scanning electron microscope was a HITACHI 4500-I apparatus fit out with a field emission gun (SEM-FEG) working at $3.0 \mathrm{kV}$. Transmission electron microscopy (TEM) was performed on HITACHI H7650 equipment with a field emissive gun, operating at $80 \mathrm{kV}$.

UV-vis-NIR absorption properties were investigated by diffuse reflectance spectroscopy using a Cary 5000E spectrophotometer equipped with an integrating sphere in the wavelength region between 200 and $2500 \mathrm{~nm}$. Samples were placed in a Suprasil1 cell equipped with a quartz window; halon standard ( $6 \mathrm{~mm}$ deep and $1 \mathrm{~g} / \mathrm{cm}^{3}$ density) was used as a white reference.

\section{Results and discussion}

\section{Mie scattering calculations}

Thanks to its efficient absorption/reflection properties in the UV and IR ranges, n-doped zinc oxide nanoparticles are promising candidates for the fabrication of visible-transparent and heat-insulating glazing. From a conceptual viewpoint, it may be envisioned two types of smart window devices able to cut the infrared range while remaining transparent in the visible one for use in glazing thermal insulation (Fig. 1b,d): dense and thin films deposited on a glass or polymer substrate, and composite panes or other systems such as varnishes and coatings added or spread to the glazing in which TCO nanoparticles are dispersed in a glass or polymeric matrix. Both systems are based on the specific dependence of the complex refractive indexes $\left(n_{m}\right.$ : real part of the complex index of refraction and $k_{m}$ : absorption coefficient) versus the light wavelength of transparent conductive oxides (TCOs). Indeed, in these materials, free electron gas (well described by the Drude model) $)^{5}$ induces drastic changes of $n_{m}$ and $k_{m}$ indexes near the plasma frequency such a way that these semiconducting oxides are transparent to light for energy higher than the critical frequency and reflective/absorbent for lower energy. In a first step, the calculation of realistic values of $n_{m}$ and $k_{m}$ versus wavelength for doped-ZnO was obtained from the series of the following equations:

$\varepsilon^{\prime}(\omega)=\varepsilon_{\infty}\left(1-\omega_{p}^{2} / \omega^{2}\right)$ and $\varepsilon^{\prime}(\omega)=\left(\varepsilon_{\infty} \omega_{p}^{2}\right) /\left(\omega^{3} \cdot \tau\right) \quad$ eq. 1

$n_{o}=\left\{\left[\left(\varepsilon^{, 2}+\varepsilon^{2}\right)^{1 / 2}+\varepsilon^{2}\right] / 2\right\}^{1 / 2}$

eq. 2

$k_{o}=\left\{\left[\left(\varepsilon^{2}+\varepsilon^{2}\right)^{1 / 2}-\varepsilon^{2}\right] / 2\right\}^{1 / 2}$

eq. 3

with $\tau=\mathrm{e} /\left(\mu, m^{*}\right)$ and where the plasma wavelength $\lambda_{p}=1$ $\mu \mathrm{m}\left(\lambda_{\mathrm{p}}=\mathrm{c} / \omega_{\mathrm{p}}\right), \mu=20 \mathrm{~cm}^{2} /(\mathrm{V} . \mathrm{s}), \varepsilon_{\infty}=4$ and the effective mass of the charge carriers $m_{c}^{*}=0.3 m_{e-\text {, }}$ i.e. standard values extracted from the literature. ${ }^{6}$

The $n_{m}$ and $k_{m}$ curves versus wavelength were plotted versus light wavelength in the $200-2500 \mathrm{~nm}$ range (Fig. 1a).

Nowadays, most of the studies have focused on the elaboration of dense and thin films for the control of the

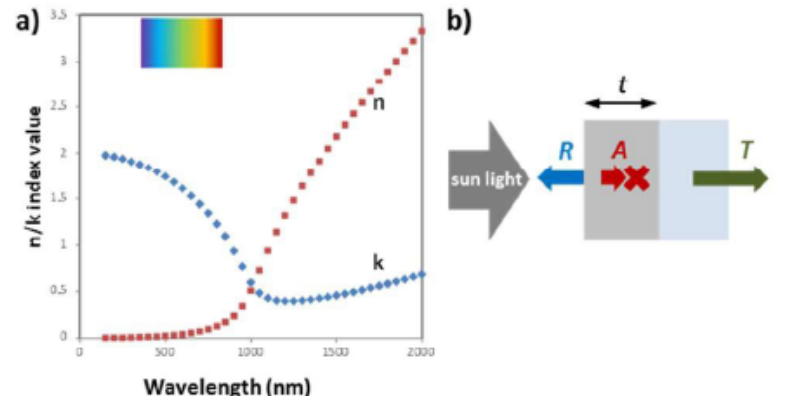

Wavelength $(\mathrm{nm})$

d)

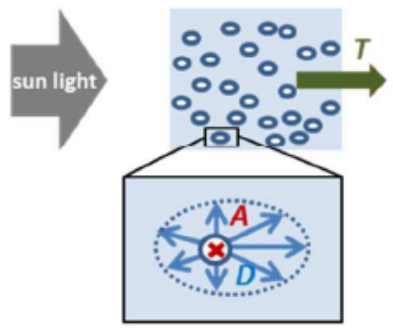

e)

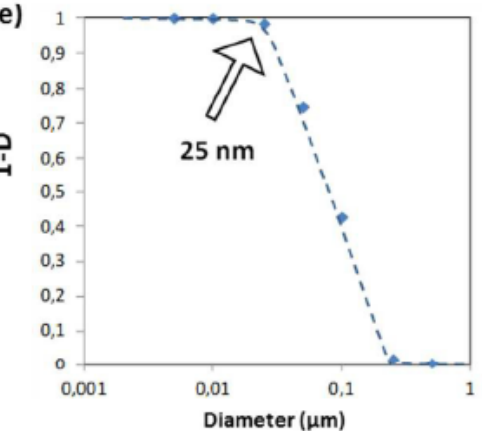

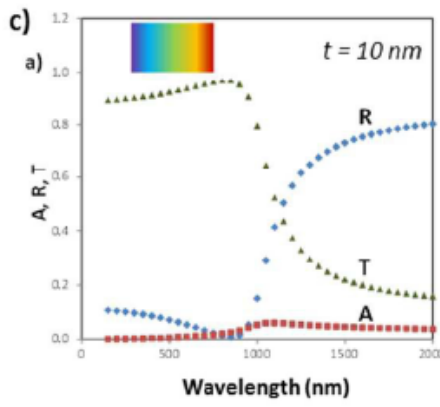

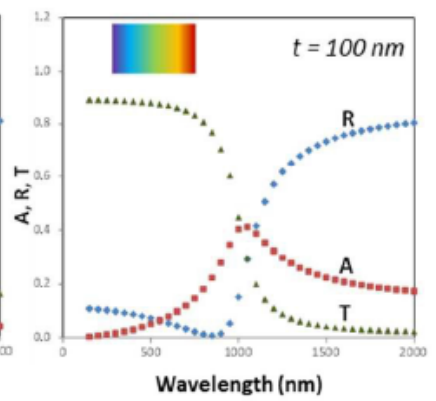

f)

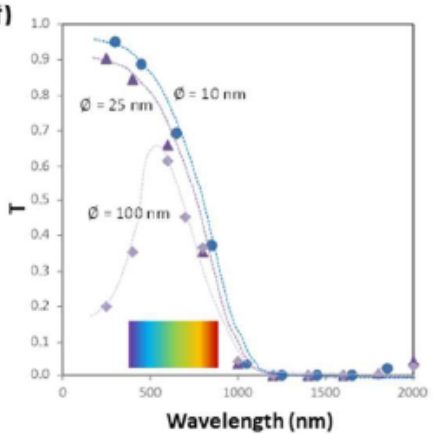

Fig. 1 a) $n$ and $k$ parameter evolution vs wavelength near the plasma frequency as computed from Eq. 1, 2 and 3; b and c) Calculations of light phenomena considering a device (b) consisting of a dense $\mathrm{ZnO}$ thin film on a non-absorbent substrate (glass or polymer) with R: reflectivity at the interface air-film, A: absorption through the film, T: transmission, for film thicknesses ( $t$ ) of 10 and $100 \mathrm{~nm}$ and using $a b$ initio refractive indexes; $d$, e and f) Calculations from a doped-ZnO nanoparticles dispersion (d) in a non-absorbent matrix (liquid, glass or polymer) with $\mathrm{D}$ scattering from the particles, $\mathrm{A}$ absorption through the particles and $\mathrm{T}$ transmission. e) Evolution of the one-direction scattering for $\lambda=400 \mathrm{~nm}$ of non- 
absorbent colloids $\left(n_{p}=2, k_{p}=0\right)$ in a glass or polymeric matrix $\left(n_{m}=1.4, k_{m}=0\right)$ versus the particles size; $f$ ) Mie calculations of the transmission of doped-ZnO particles (with ab initio refractive indexes) dispersed in a glass or polymer $\left(n_{m}=1.4, k_{m}=0\right)$ versus light wavelength for various particles diameters of 100,25 and $10 \mathrm{~nm}$.

infrared/visible transmission through windows. These films of doped-zinc oxide, for instance, aluminum-doped (AZO), gallium-doped (GZO), fluorine-doped zinc oxide (FZO) or tindoped indium oxide (ITO) are fabricated from physical deposition processes achieving dense and non-diffusive films with thickness about $10-100 \mathrm{~nm}$. In such thin films, the target is to get a transparent film for visible range and an opaque film in infrared range by relying mainly on the reflective properties of the films. It means that the films being easily prepared dense and so non-diffusive, the optical properties of the display may only be optimized through the control of the balance of the three optical phenomenon components: transmission, absorption and reflection trying to be thinner as possible to limit the absorption, especially in the visible range, without degradation of the conductivity to produce an intense reflection of the IR rays. This kind of films is being extensively studied and their reflectance-absorption-transmission curves on the $200-2500 \mathrm{~nm}$ wavelength range are abundantly reported. ${ }^{7}$ In order to check the soundness of the $n_{o}$ and $k_{o}$ values previously proposed, we performed an $a b$ initio calculation of the properties of such thin films (with thickness equal to 10 or $100 \mathrm{~nm}$ ) for which the scattering is null (as considered perfectly dense) and considering that the reflection at the substrate-air interface and at the film-substrate interface is negligible in comparison with the reflection at the film-air interface. With these first approximations, the total transmission $(T)$ versus the light wavelength of the display can be roughly deduced from the consideration that $R+A+T=1$ and the two following equations describing the reflectivity $(R)$ at the air-film interface (eq. 4) and the absorption (A) through the thickness (I) of the semi-conducting film (eq. 5) :

$R=\left[\left(n_{o}-1\right)^{2}+k_{o}^{2}\right] /\left[\left(n_{o}+1\right)^{2}+k_{o}^{2}\right]$

$\mathrm{A}=(1-\mathrm{R}) \times\left[1-\exp \left(-4 \pi k_{o} l / \lambda\right)\right]$

eq. 4

The A, R, T curves obtained (Fig. 1c) are consistent to the experimental results reported in the literature, ${ }^{8}$ showing that the proposed values of $n_{o}$ and $k_{o}$ refractive indexes are realistic.

However, reports of the same three experimental or theoretically-calculated curves for a composite medium in which nanoparticles are embedded in a transparent matrix are missing. In order to validate the ability of this $2^{\text {nd }}$ kind of devices to transmit the visible rays while absorbing the infrared ones, the free soft Mie calculator was used on predefined composite systems. ${ }^{\ddagger}$ In such composite panes, there is no reflection at the matrix-air interface, or a very limited one considering the index of refraction of the matrix is quite low ( $n_{m}$ about 1.5) and its absorbing coefficient is null $\left(k_{m}=0\right)$. However, the scattering phenomenon occurring at every nanoparticle-matrix interface should limit drastically the transmission. Hence, in such a composite device, the limiting factor to the transmission is the scattering, and it should be circumvented by limiting the particle sizes and/or the difference between the refractive indexes of the nanoparticles and the matrix. The scattering intensity at the nanoparticlematrix interfaces is indeed well defined in the Rayleigh regime as:

$$
\begin{aligned}
I_{s}= & I_{0} \times\left(8 \pi^{4} a^{6}\right) /\left(2 r^{2} \lambda^{4}\right) \times\left[\left(m^{2}-1\right) /\left(m^{2}+1\right)\right]^{2} \\
& \times\left(1+\cos ^{2} \theta\right)
\end{aligned}
$$

with $m=n_{o} / n_{m}, a$ the nanoparticle size, $r$ the distance of the measurement from the diffusive center, and $\theta$ the angle between the incident and scattering light beams. In a first step, in order to simulate the influence of the nanoparticle size on the scattering intensity in the visible range, a calculation of the scattering for $\lambda=400 \mathrm{~nm}$ considering non-absorbent colloids with $n_{o}=2, k_{o}=0$ in a liquid, glass or polymeric matrix with $n_{m}=1.4$ and $k_{m}=0$ for various particle sizes was performed (Fig. 1e). The calculation was made on a composite medium whose total thickness of $\mathrm{ZnO}$ to be crossed through is $500 \mathrm{~nm}$. From this calculation, it can be seen that the value of $25 \mathrm{~nm}$ appears to be the critical particle size to not be exceeded for preserving the visible-transparency. In a second step, Mie calculations of the transmission of doped-ZnO particles (using the ab initio refraction indexes) dispersed in a glass or polymer $(\mathrm{nm}=1.4, \mathrm{~km}=0)$ versus the light wavelength for particles with diameters of $100 \mathrm{~nm}, 25 \mathrm{~nm}$ and $10 \mathrm{~nm}$ were performed (Fig. 1f). As previously, the calculation was made considering the total thickness of $\mathrm{ZnO}$ was $500 \mathrm{~nm}$. From this last figure, it can be deduced that the optical properties of composite with particle size of $25 \mathrm{~nm}$ should be optimal, since the IR transmission at $2000 \mathrm{~nm}$ would be near null whereas the transmission at $400 \mathrm{~nm}$ (in the UV-visible range frontier) would remain about $90 \%$. Furthermore, the gain in visible transmission decreasing the grain size from 25 to $10 \mathrm{~nm}$ is negligible. Moreover the increasing of the particle size from 25 $\mathrm{nm}$ to $100 \mathrm{~nm}$ deteriorates dramatically the transmission, and even more so that wavelengths are short: the transmission at $400 \mathrm{~nm}$ falls in the value of about $40 \%$ for $100 \mathrm{~nm}$ diameter particles.

\section{Preparation of transparent ZnO:Ga nanoparticle dispersions}

We recently reported that the polyol synthesis route developed by Fievet and coll. ${ }^{9}$ known to give micron-scaled supracolloidal ZnO particles (SPC) made of aggregates of primary crystallites (Fig. 2a) was compatible for the aliovalent doping with $\mathrm{Ga}^{3+}$ cation. $^{2}$ The size of the crystallites 

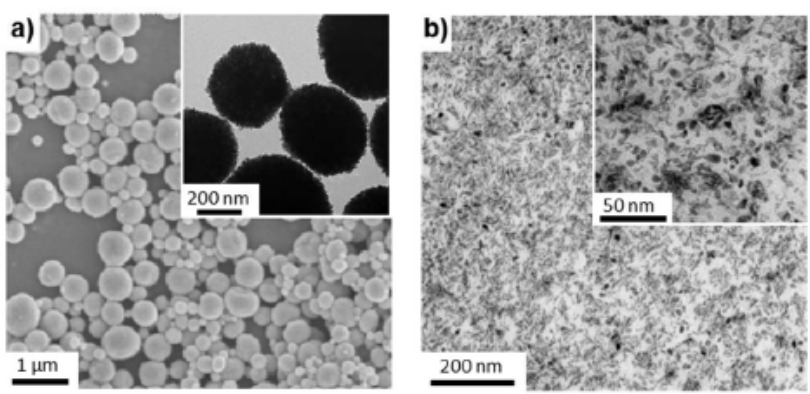

Fig. 2 a) SEM and TEM (in insert) micrographs of the pristine $\mathrm{ZnO}: \mathrm{Ga}$ resulting from polyol synthesis; b) TEM micrographs of the ZnO:Ga colloids obtained after disaggregation using HDT and ultrasonication.

constituting the SPC are of about $10 \mathrm{~nm}$ in the 110 direction and slightly smaller dimensions in the other ones. Experiments by diffuse reflectance spectroscopy showed that the asobtained SPC are efficient selective IR-absorbers (up to $90 \%$ ) especially for a gallium concentration of about $2.3 \%$. Nevertheless, these particles are too large and scatter visible light even when they are dispersed in liquid media. Therefore the dispersion was not transparent preventing these particles to fulfil the requirements for glazing application. Several attempts for disaggregating these crystallites, e.g. by ultrasonication, remained unsuccessful. As a consequence, we investigated the possibility for simultaneously forcing the disaggregation of the SPC while stabilizing the dispersions of the released primary crystallites. For this, we used a strong ligand capable to exchange the weaker ones, such as DEG molecules adsorbed on $\mathrm{ZnO}$ and involved in the cohesion of the nanocrystallites. The exchange ability among different ligands decreases in an order of thiols, carboxylic acids, amines, and alcohols. So we used hexadecane-1-thiol (HDT) so that the surface coverage with the long alkyl tails enables the crystallite solubility in nonpolar solvents or halogenoalkanes such as chloroform.
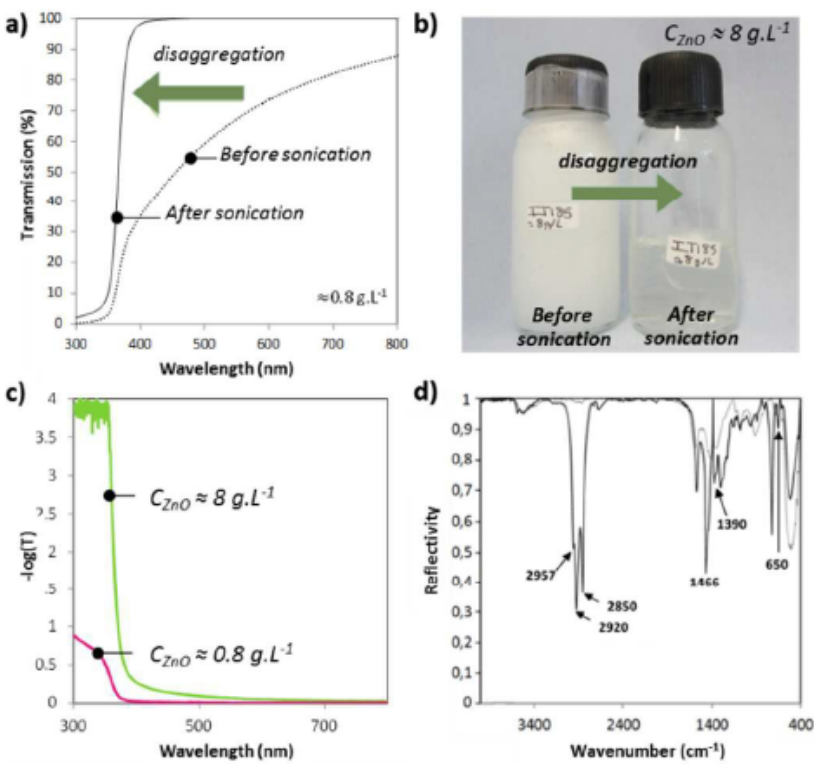

Fig. 3 a) UV-Vis transmission spectra after the 10 -fold dilution in $\mathrm{CHCl}_{3}\left(08 \mathrm{~g} \cdot \mathrm{L}^{-1}\right)$ before and after ultrasonication; b) Picture of the $8 \mathrm{~g} . \mathrm{L}^{-1}$ dispersions before and after a 20-min ultrasonication stage; c) Variation vs wavelength of the pseudo-absorbance of the HDTmodified $\mathrm{ZnO}$ nanoparticles redispersed in chloroform at 0.8 or $8 \mathrm{~g} \cdot \mathrm{L}^{-1}$ concentration; d) FTIR spectra (after background correction) of a ZnO-HDT sample (black line) and comparison with a pristine $\mathrm{ZnO}$ sample (dotted line).

HDT molecules were put in contact with the $\mathrm{ZnO}$ particles in DEG in a post-synthesis stage, using ethanol as a co-solvent. Knowing that the calculated area per alkanethiolate molecule assembled to a hexagonal monolayer on Au (111) surface, ${ }^{10}$ is $21.4 \AA^{2}$ and assuming that the specific surface area of the fully disaggregated $\mathrm{ZnO}$ particles is about $105 \mathrm{~m}^{2} / \mathrm{g}$ (considering in first approximation, a spherical crystallite of $10 \mathrm{~nm}$-diameter), we calculated the minimal concentration of HDT to be added for getting a surface monolayer, called $[\mathrm{HDT}]_{\text {monolayer }}$ In this first experiment an excess of 20 was used. The disaggregation occurred when the modified nanoparticles were immersed in $\mathrm{CHCl}_{3}\left(\mathrm{n}=1.45\right.$ at $20^{\circ} \mathrm{C}$ ) with the help of the sonication to break the dipolar interactions (london's type) responsible of the NP cohesion and to favour the solvation of the hydrophobic tails of the ligands. It appeared that visible transparent dispersions at a weight concentration of $0.8 \mathrm{~g} . \mathrm{L}^{-1}$, for instance, were obtained at the expense of several-minutes treatment in sonication bath as demonstrated by recording the UV-visible transmission spectra (Fig. 3a). The average transmission in the visible range of the dispersion increased, from about 60 to $100 \%$ at $500 \mathrm{~nm}$ for this dispersion after sonication. The photograph in Fig. 3b shows dispersions (8 g. $\mathrm{L}^{-}$ ${ }^{1}$ ) before and after sonication: the milky (turbid) $\mathrm{ZnO}$ dispersion became highly transparent. The absorbance spectra recorded after sonication (Fig. 3c) exhibit not only a very low scattering at $500 \mathrm{~nm}$ but also a very high absorbance at 340 $\mathrm{nm}$ related to the bandgap of $\mathrm{ZnO}$, in comparison with dispersions before ultrasonication application. This observation is still true whatever the $\mathrm{ZnO}$ concentration, e.g. 8 or $0.8 \mathrm{~g} \cdot \mathrm{L}^{-1}$. 

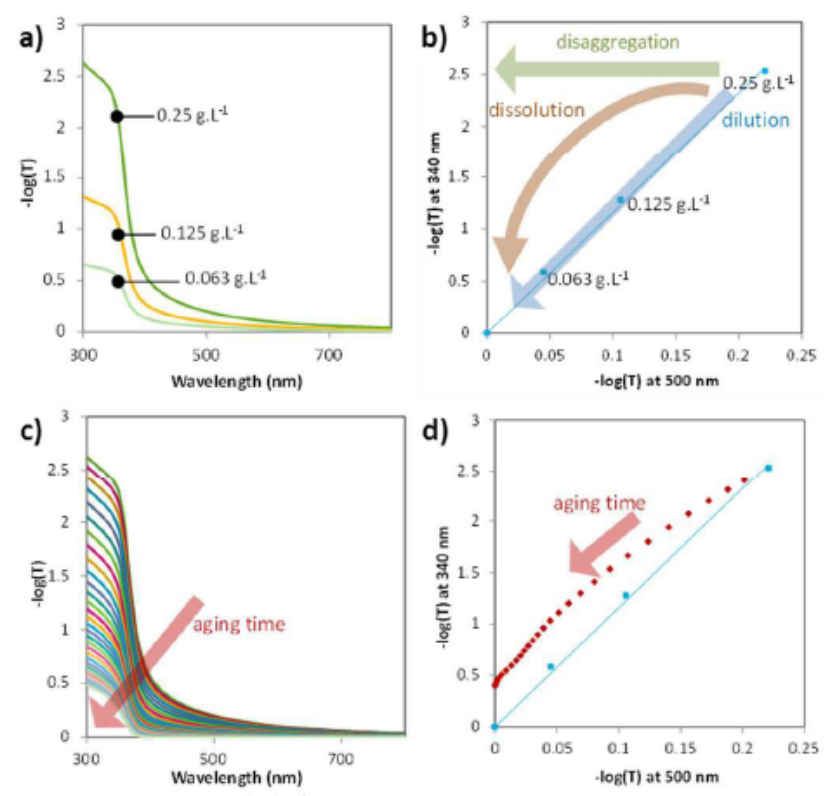

Fig. 4 a) Variation vs. wavelength of the pseudo-absorbance of DEG-based ZnO SPC dispersions at different weight concentrations; b) Master curve derived from these spectra and expected evolutions vs. time of the disaggregation and dissolution phenomena; c) Variation vs wavelength of the pseudo-absorbance of acetate-modified $\mathrm{ZnO}$ aggregates dispersed in DEG ( $\left.025 \mathrm{~g} . \mathrm{L}^{-1}\right)$ versus time, the spectra having been recorded every 6 min.; d) Evolution of absorbance and diffusion and its comparison to the master curve.

We also checked that the desopacification of the dispersions resulted from a disaggregation process and not from a dissolution one by using acetic acid as positive control. Master curves were obtained by graphing the absorbance of DEG-based ZnO aggregate dispersions at $340 \mathrm{~nm}$ $\left(A_{340}=-\log T_{340}\right) v s$. their diffusion at $500 \mathrm{~nm}$ (calculated as a pseudo-absorbance: $D_{500}=-\log \mathrm{T}_{500}$ ) for different $\mathrm{ZnO}$ concentrations (Fig. 4a,b). It may be observed that they are straights passing through the origin and illustrate a dilution phenomenon when it is considered from the top towards the bottom (blue arrow), meaning that both diffusion at $500 \mathrm{~nm}$ and the phenomenon due to the band gap at $300 \mathrm{~nm}$ are proportional to the particles concentration. As a consequence, a disaggregation process should lead to variations as illustrated by the green arrow, illustrating that the absorption component is preserved contrarily to the diffusion one. Lastly, a dissolution process (red arrow) should represent a situation intermediary between both previous ones because occur simultaneously the decrease of the amount of $\mathrm{ZnO}$ particles, i.e. the absorbance at $340 \mathrm{~nm}$, and the decrease of the size of the aggregates, i.e. the absorbance at $500 \mathrm{~nm}$ but in a lesser extent than in the dilution situation. Therefore recording the UV/visible spectra as a function of time should give insights into the nature of the phenomenon leading to the dispersion transparency. The spontaneous desopacification of the dispersion of the acetate-treated Ga-doped $\mathrm{ZnO}$ particles in every $6 \mathrm{~min}$ (Fig. 4c,d). It may be observed that the absorbance decreased simultaneously at the 340 and $500 \mathrm{~nm}$ wavelengths demonstrating a progressively slowed down dissolution phenomenon. On the contrary, in the case of the HDTmodified particles, the desopacification of the dispersion results from a nearly pure disaggregation phenomenon. The success of the disaggregation strategy by dispersing the HDTmodified nanoparticles in chloroform $\mathrm{CHCl}_{3}$ was checked by different ways. Firstly, the absorbance curves recorded after ultrasonication of the dispersions exhibit very low diffusion at $500 \mathrm{~nm}$ combined to a very high absorbance at $340 \mathrm{~nm}$ in comparison with all the previous dispersions or the same dispersions before ultrasonication (Fig. 3c). This observation remains true whatever the weight concentration, e.g. 8 or 0.8 g. $\mathrm{L}^{-1}$. It appeared that visible transparent dispersions (with 0.8 g. $\mathrm{L}^{-1}$ ) were obtained at the expense of several-minutes sonication as demonstrated by recording the UV-visible transmission spectra (Fig. 3a). The average transmission in the visible range increased from 70 to $100 \%$ after sonication.

The TEM images of the HDT-modified Ga-doped $\mathrm{ZnO}$ particles after ultrasonication (Fig. 2b) confirm the disaggregation of the particles. They clearly show the disappearance of the aggregates and the primary crystallites nicely spread onto the carbon film of the TEM grid. Furthermore, X-ray diffraction analyses were performed on the dispersed particles after extraction from the liquid phase using high rotational speed centrifugation. The patterns reported (Fig. 5) shows that every peak can be indexed in the ZnO-würtzite phase, at the exception of a low-angle and very large peak which would be the signature of the structuration of the particles linked by the HDT coated layers ("colloidal crystal" formation). Scherrer calculation was made from the peak width (FWHM: Full Width at Half Maximum) on three of the main peaks, leading to an average crystallite size of about 
11-12 $\mathrm{nm}$. This value is in perfect agreement with the previous TEM observations.
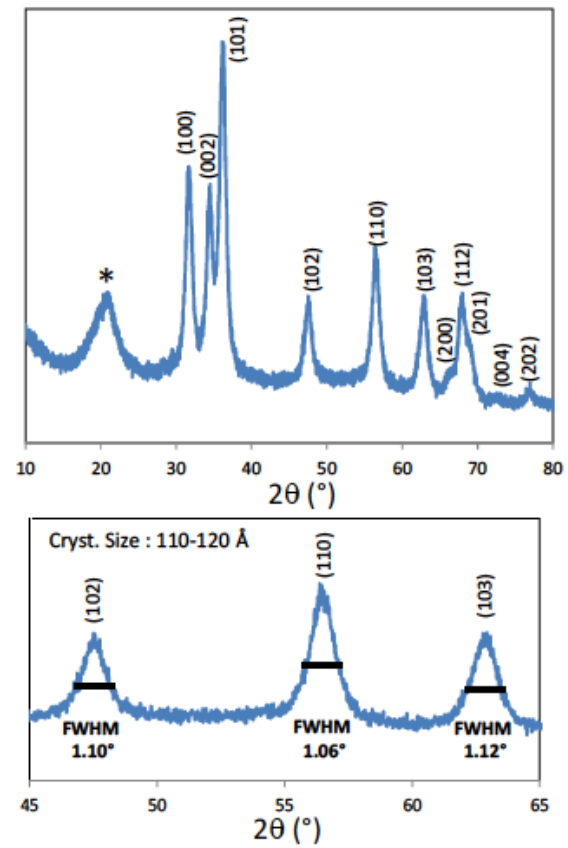

Fig. $5 \mathrm{X}$-ray diffraction patterns of the dispersed particles after extraction from the liquid phase using high rotational speed centrifugation. Bottom: focus on the peak width.

The chemical signature of HDT ligands grafted onto the $\mathrm{ZnO}$ particle surface was evidenced by IR spectroscopy in diffuse reflectance mode on dried particles (Fig. 3d). The strong IR bands at 2920 and $1390 \mathrm{~cm}^{-1}$ were assigned to the asymmetrical and symmetrical (rocking and twisting) stretching modes of the alkyl chains $\left(\mathrm{CH}_{2}\right)$, respectively, while the band at $1466 \mathrm{~cm}^{-1}$ was attributed to deformation vibration $\left(\delta \mathrm{CH}_{2}\right) .{ }^{11}$ Moreover, the bands observed at 2957 and $2850 \mathrm{~cm}^{-1}$ were assigned to the asymmetrical and symmetrical (rocking and twisting) stretching modes of the methyl groups $\left(\mathrm{CH}_{3}\right)$. All these band wavelengths are close to those observed for gold or $\mathrm{ZnO}$ nanoparticles capped with alkanethiol molecules. ${ }^{12}$ At last, the vibration bands observed at 510 and $650 \mathrm{~cm}^{-1}$ were assigned to $\mathrm{Zn}-\mathrm{O}$ and $\mathrm{Zn}-\mathrm{S}$ bonds. ${ }^{13}$
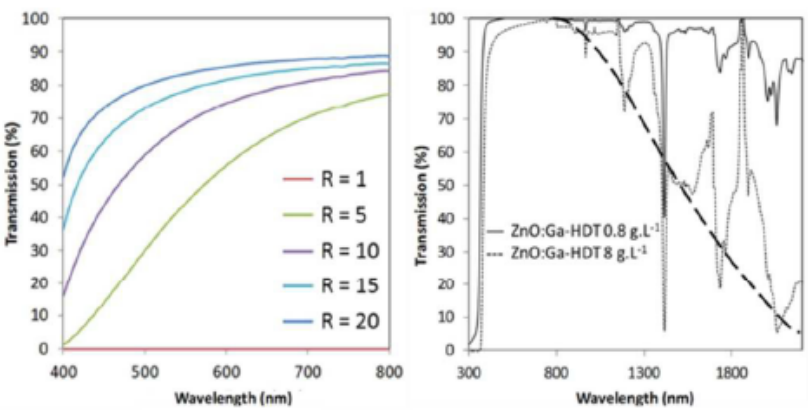

Fig. 6 a) Visible spectra of $\mathrm{ZnO}-\mathrm{HDT}$ dispersions in $\mathrm{CHCl}_{3}$ prepared with different HDT concentrations and after ultrasound application; b) UV-Vis-NIR transmittance spectra of $\mathrm{ZnO}-\mathrm{HDT} / \mathrm{CHCl}_{3}$ dispersions with concentrations of $0.8 \mathrm{~g} \cdot \mathrm{L}^{-1}$ and $8 \mathrm{~g} \cdot \mathrm{L}^{-1}$. The dashed line is just a guide for the eye.

The issue of the amount of HDT to add in order to get an efficient desopacification of the dispersion was also addressed. The $[\mathrm{HDT}]_{\text {monolayer }}$ value equal (to $6.5 \mathrm{mM}$ in these conditions for a final $\mathrm{ZnO}$ concentration of $2.67 \mathrm{~g} / \mathrm{L}$ ) was fixed as the reference and we prepared several dispersions in $\mathrm{CHCl} 3$ for different [HDT] values such as the ratio $\mathrm{R}=$ [HDT]/[HDT]monolayer was equal to 1, 5, 10, 15 and 20 (Fig. 6a). Ultrasonication was performed until the UV-visible spectra were stabilized (around $15 \mathrm{~min}$ ). It was observed that the dispersions remained turbid up to [HDT]/[HDT]monolayer $=10$ and that an excess of 20 was necessary for reaching a transparency of $82 \%$ (Fig. 6a). The exchange kinetics and the diffusion of the alkanethiol molecules between the nanocrystallites were improved by a better mass transport of ligands close to the $\mathrm{ZnO}$ surface which occurs for higher concentrations. Fig. $6 \mathrm{~b}$ shows UV-Vis-NIR transmittance spectra of $\mathrm{ZnO}-\mathrm{HDT} / \mathrm{CHCl}_{3}$ dispersions. It is clearly observed that the transmission window in the visible region is framed by two absorbent barriers in the UV and IR ranges, as expected. For this last one, the subtraction of the solvent contribution is rather difficult due to the presence of HDT ligands which cannot be subtracted. Nevertheless, the dashed line shows that the transmission tends to decrease in the IR region.

\section{Conclusions}

Transparent-visible and UV/IR-opaque dispersions were achieved by disaggregation of Ga-doped ZnO SPC obtained through the polyol synthesis route. The optical behavior of these dispersions validates the preliminary modelling based on Mie scattering calculations. It may be emphasized that alkanethiol molecules such as hexadecane-1-thiol are efficient ligands for stabilizing dispersions of Ga-doped $\mathrm{ZnO}$ nanocrystallites in organic solvents. This study may be regarded as a starting point for further developments consisting in dispersing such nanoparticles in visibletransparent matrices such as organic glasses made of poly(methyl methacrylate) for instance, or varnishes in order to move to usable devices.

\section{Acknowledgements}

We acknowledge financial support from The French National Research Agency (CITOYEN project, contract \# ANR A2010 JCJC $080501)$.

\section{Notes and references}

¥ S. Prahl, Mie scattering calculator. Software available: http://omlc.org/calc/mie calc.htm|

1 K. Y. Cheong, N. Muti and S. R. Ramanan, Thin Solid Films, 2002, 410, 142; H. Gomez, A. Maldonado, R. CastanedoPerez, G. Torres-Delgado and M. de la Olvera, Mater. 
Charact., 2007, 58, 708; M. Suguyama, A. Murayama, T. Imao, K. Saiki, H. Nakanishi and S.F. Chichibu, Phys. Status Solidi A-Appl. Mater., 2006, 203, 2882; S. Major, A. Banerjee and K.L. Chopra, Thin Solid Films, 1984, 122, 31-43; W.W. Wenas, A. Yamada, K. Takahashi, M. Yoshinoa and M. Konogai, J. Appl. Phys., 1991, 77, 107.

$2 \mathrm{H}$. Sérier, M. Gaudon and M. Ménétrier, Solid State Sci., 2009, 11, 1192 ; H. Sérier, A. Demourgues and M. Gaudon, Inorg. Chem., 2010, 49, 6853; I. Trenque, S. Mornet, E. Duguet and M. Gaudon, MRS Bull., 2013, 48, 1155; I. Trenque, S. Mornet, E. Duguet and M. Gaudon, Inorg. Chem., 2013, 52, 12811; I. Trenque, S. Mornet, E. Duguet, J. Majimel, A. Brüll, K. Teinz, E. Kemnitz and M. Gaudon, Opt. Mater., 2013, 35, 661.

3 B. Lewis and D. Paine, MRS Bull., 2000, 25, 22; I. Hamberg and C.G. Granqvist, Solar Energy Mater., 1984, 11, 239; A Hjortsberg, I. Hamberg and C.G. Granqvist, Thin Solid Films, 1982, 90, 323; R.P. Howson, J.N. Avaritsiotis, M.I. Ridge and C.A. Bishop, Thin Solid Films, 1979, 63, 163; C.M. Lampert, Solar Energy Mater., 1981, 6, 1; T. Coutts, D. Young and X. Li, MRS Bull., 2000, 25, 58; R.G. Gordon, MRS Bull., 2000, 25, 52.

4 W. Tang and D. Cameron, Thin Solid Films, 1994, 238, 83; M. Hilgendorff, L. Spanhel, C. Rothenhäusler and G. Muller, J. Electrochem. Soc., 1998, 145, 3632; R. Buonsanti, A. Llordes, S. Aloni, B. Helms and D. Milliron, Nano Lett., 2011, 11, 4706.

5 P. Drude, Ann. Phys. (Berlin), 1900, 306, 566 ; P. Drude, Ann. Phys. (Berlin), 1900, 308, 369.

6 I. Saadeddin, Ph.D. dissertation, Université Bordeaux 1, 2007.

7 S. Calnan and A.N. Tiwari, Thin Solid Films, 2010, 518, 1839; P.D.C. King and T.D. Veal, J. Phys.: Condens. Matter, 2000, 23, 334214; K. Ellmer and T. Welzel, J. Mater. Res., 2012, 27, 765; C.G. Granqvist, Appl. Phys. A, 1993, 57, 19; E.L. Runnerstrom, A. Llordés, S.D. Lounis and D.J. Milliron, Chem. Commun., 2014, 50, 10555.

8 K. Maki, N. Komiya and A. Suzuki, Thin Solid Films, 2003, 445, 224; I. Hamberg, A. Hjortsberg and C. Granqvist, Appl. Phys. Lett., 1985, 40, 362.

9 D. Jezequel, J. Guenot, N. Jouini and F. Fievet, Mater. Sci. Forum, 1994, 339, 152; P. Toneguzzo, G. Viau, O. Acher, F. Fiévet-Vincent and F. Fiévet, Adv. Mater., 1998, 10, 1032; G. Viau, J. Y. Piquemal, M. Esparrica, D. Ung, N. Chakroune, F. Warmont and F. Fiévet, Chem. Commun., 2003, 9, 2216.

$10 \mathrm{H}$. Sellers, A. Ulman, Y. Shnidman and J. E. Eilers, J. Am. Chem. Soc., 1993, 115, 9389.

11 B. Smith, Infrared Spectral Interpretation. A Systematic Approach, C. P. CRC Press, Ed., ISBN 0-8493-2463-7, 1999

12 . L. Chen, J. Xu, J. D. Holmes and M. A. Morris, J. Phys. Chem. C 2010, 114, 2003; M. Porter, T. Bright, D. Allara and C. Chidsey, J. Am. Chem. Soc., 1987, 109, 3559.

13 V. Mote, V. Huse and B. Dole, AIP Conf. Proc., 2013, 1512, 188. 
ZnO:Ga supracolloidal particles are disaggregated into transparent dispersions in the visible range and may be used as an effective UV and infrared filter.
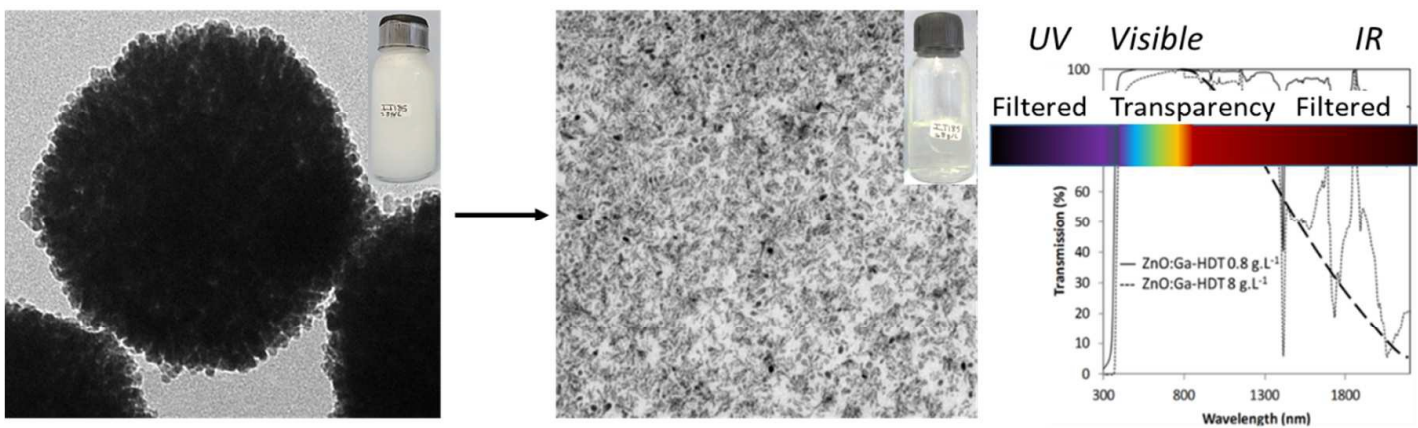